Rev. Biol. Trop. 52(1): 27-30, 2004

www.ucr.ac.cr www.ots.ac.cr www.ots.duke.edu

NOTA

\title{
Una nueva especie de Euglena (Euglenozoa: Euglenales) aislada de ambientes extremófilos en las Pailas de Barro del Volcán Rincón de la Vieja, Costa Rica
}

\author{
Ana Sittenfeld ${ }^{1}$, Maribelle Vargas $^{3}$, Ethel Sánchez ${ }^{3}$, Marielos Mora $^{1}$ \& Aurelio Serrano ${ }^{2}$ \\ 1 Centro de Investigación en Biología Celular y Molecular (CIBCM), Universidad de Costa Rica, Ciudad Universitaria \\ Rodrigo Facio, 2060, San José, Costa Rica. \\ 2 Instituto de Bioquímica Vegetal y Fotosíntesis (IBVF), Universidad de Sevilla y CSIC, Américo Vespucio sln, 41092 , \\ Sevilla, España. \\ 3 Centro de Investigación en Estructuras Microscópicas, Universidad de Costa Rica, Ciudad Universitaria Rodrigo \\ Facio, San José, Costa Rica. \\ Corresponding autor: sitten@ racsa.co.cr
}

Recibido 02-III-2002. C Corregido 22-III-2003. Aceptado 23-IV-2003.

\begin{abstract}
A new species of euglena isolated from a hot and acid mud pool located in Las Pailas de Barro, Volcán Rincón de la Vieja, Costa Rica is described. This species inhabits hot and acid environments. Euglena pailasensis sp. nov. main features are: the absence of flagella, the presence filaments like "pilis", the presence of chloroplasts with pyrenoids crossed by several tylakoids, and acid and heat tolerance. Molecular phylogeny studies using $18 \mathrm{~S}$ rDNA and Gap $C$ genes indicated that the new species is related to E mutabilis. Its taxonomic characters based on morphology, biology and sequence of the $18 \mathrm{~S}$ rDNA and Gap $C$ genes are discussed and compared with other closely related species of the genus.
\end{abstract}

Key words: Protozoa, Euglena, acidic hot mud pool, thermo-tolerance, $18 \mathrm{~S}$ rDNA, GapC.

Las euglenas son protozoos (Phylum Euglenozoa, Orden Euglenales) caracterizados por presentar una cubierta celular o película, algunas especies presentan en sus cloroplastos pirenoides atravesados por varios tilacoides, manchas oculares y en general se mueven mediante flagelos; también presentan movimiento euglenoide conocido como metabolia, que involucra cambios de forma. La película está integrada por la membrana plasmática y por bandas proteicas flexibles dispuestas en forma helicoidal conocidas como mionemas (Vismara et al. 2000). Los cloroplastos pueden ser discoidales, en forma de escudo o de cinta, morfotipos que se asocian con diferentes especies.

La mayoría de las especies de euglenas habitan en agua dulce, aunque algunas son de agua salada. Recientemente informamos del hallazgo de un euglenoide que habita la superficie de las Pailas de Barro Caliente, sector Las Pailas, Volcán Rincón de la Vieja, Costa Rica (Sittenfeld et al. 2002). El objetivo de esta comunicación es hacer la descripción formal de tal euglenoide como nueva especie, ya que en dicho artículo (Sittenfeld et al. 2002) se usó un nombre sin descripción (nomem nudum).

\section{DESCRIPCIÓN}

\section{Euglena pailasensis nov. sp. (Euglenozoa: Euglenales)}

Su tamaño es de 30-45 $\mu \mathrm{m}$ de largo y 8$10 \mu \mathrm{m}$ de ancho, presenta de 40 a 90 mionemas, cuyo grosor aproximado es de 0.8 a 


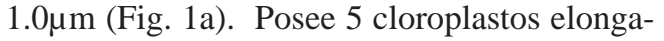
dos de 1 a $2 \mu \mathrm{m}$ de diámetro por 6 a $12 \mu \mathrm{m}$ de largo, los cuales poseen un pirenoide atravesado por varios tilacoides. Puede contener hasta 75 pequeños gránulos de paramilón citoplasmáticos, de forma bacilar y discoidal (Fig. 1b). A diferencia de otras especies del género, a E. pailasensis nov. sp no se le observan flagelos ni estructuras asociadas a éstos. De cada mionema se proyecta un filamento que en conjunto le brindan un aspecto piloso al protozoo (Fig. 1c). Además, posee termotolerancia a temperaturas cercanas a $\operatorname{los} 45^{\circ} \mathrm{C}$ y viabilidad por varias horas a $50^{\circ} \mathrm{C}$ en el sitio de colecta. Esta especie es termoluminiscente. Las secuencias del gen ADNr 18S (depositada en el GenBank: número de acceso AY029278) y del gen para la enzima Gap C (gliceraldehido-3fostato deshidrogenasa; depositada en el EMBL/GenBank: número de acceso AJ312953), son diferentes a todas las homólogas previamente descritas.

Holotipo: se encuentra depositado bajo el catálogo número UCRMZ 1-01-04 en el Museo de Zoología de la Universidad de Costa Rica, San José, Costa Rica.

Hábitat: superficie del lodo de las Pailas de Barro Caliente, Sector Las Pailas, Volcán Rincón de la Vieja, Area de Conservación Guanacaste, 24 km NNE de la ciudad de Liberia, Costa Rica. La temperatura del lodo de las Pailas de Barro varía entre 35 y $98^{\circ} \mathrm{C}$ y el pH entre 2 y 4.

Etimología: La nueva especie recibe el nombre en referencia a su sitio de aislamiento en las Pailas del Volcán Rincón de la Vieja, Costa Rica.

Discusión: Una detallada descripción ultraestructural de Euglena pailasensis es presentada en este número por Sánchez et al. 2003. Ensayos sobre la capacidad de producir termoluminiscencia, mostraron que la emisión de luz fue $8^{\circ} \mathrm{C}$ más alta que la observada para la cepa control de E. gracilis. El análisis filogenético del gen ADNr 18S indicó que la nueva especie está relacionada con E. mutabilis, con la cual comparte un $51.1 \%$ de similitud. Ambas especies tienen en común la capacidad de desarrollarse en medios muy ácidos; pero E. pailasensis puede habitar en sitios ácidos y calientes, en tanto E. mutabilis no es termoresistente. Adicionalmente, la secuencia del gen para la enzima Gap C (GAPDHC, EC 1.2.2.12), mostró un $70 \%$ de identidad con el gen para Gap C de E. gracilis (Sittenfeld et al. 2002).

\section{ENGLISH DESCRIPTION}

Euglena pailasensis nov. sp. size is 30$45 \mu \mathrm{m}$ long and $8-10 \mu \mathrm{m}$ wide. Forty to 90 myonems or pellicle strips having a thickness of $0.8-1.0 \mu \mathrm{m}$ are present over the entire cell surface (Fig. 1a). It have 5 elongated chloroplasts with varying size of ( 1 to $2 \mu \mathrm{m}$ in diameter, 6 to $12 \mu \mathrm{m}$ long). The chloroplasts contain pyrenoids penetrated by several tylakoids usually arranged in pairs. Inside the cytoplasm up to 75 paramilon grains having bacillary and discoid shapes are present (Fig. 1b). Neither flagella nor basal bodies were observed. Around the external surface, the presence of filament like structures originating from the pellicle strips, gives the euglenoid a hairy appearance (Fig. 1c). A detailed ultrastructural description is offered by Sánchez et al. (2003). The species exhibits thermo tolerance to temperatures close to $45^{\circ} \mathrm{C}$ and viability during several hours at $50^{\circ} \mathrm{C}$ at the collecting site. Thermo luminescence studies indicate an emission temperature band peaking at $8^{\circ} \mathrm{C}$ higher than that observed for the control strain of E. gracilis SAG 5/15. Molecular phylogeny studies using 18S rDNA indicated that the euglenoid is closely related to E. mutabilis, showing a $51.1 \%$ similarity at the DNA level. The sequence of the $18 \mathrm{~S}$ rDNA was deposited at GenBank with the accession number AY029278. Both species have in common the capacity to inhabit acid environments; but $E$. pailasensis inhabits acids and hot volcanic enviroment and E. mutabilis shows no thermo tolerance. In addition, the sequence for the GapC gene (glyceraldehyde-3-phosphate dehydrogenase; GAPDHC, EC 1.2.2.12) 

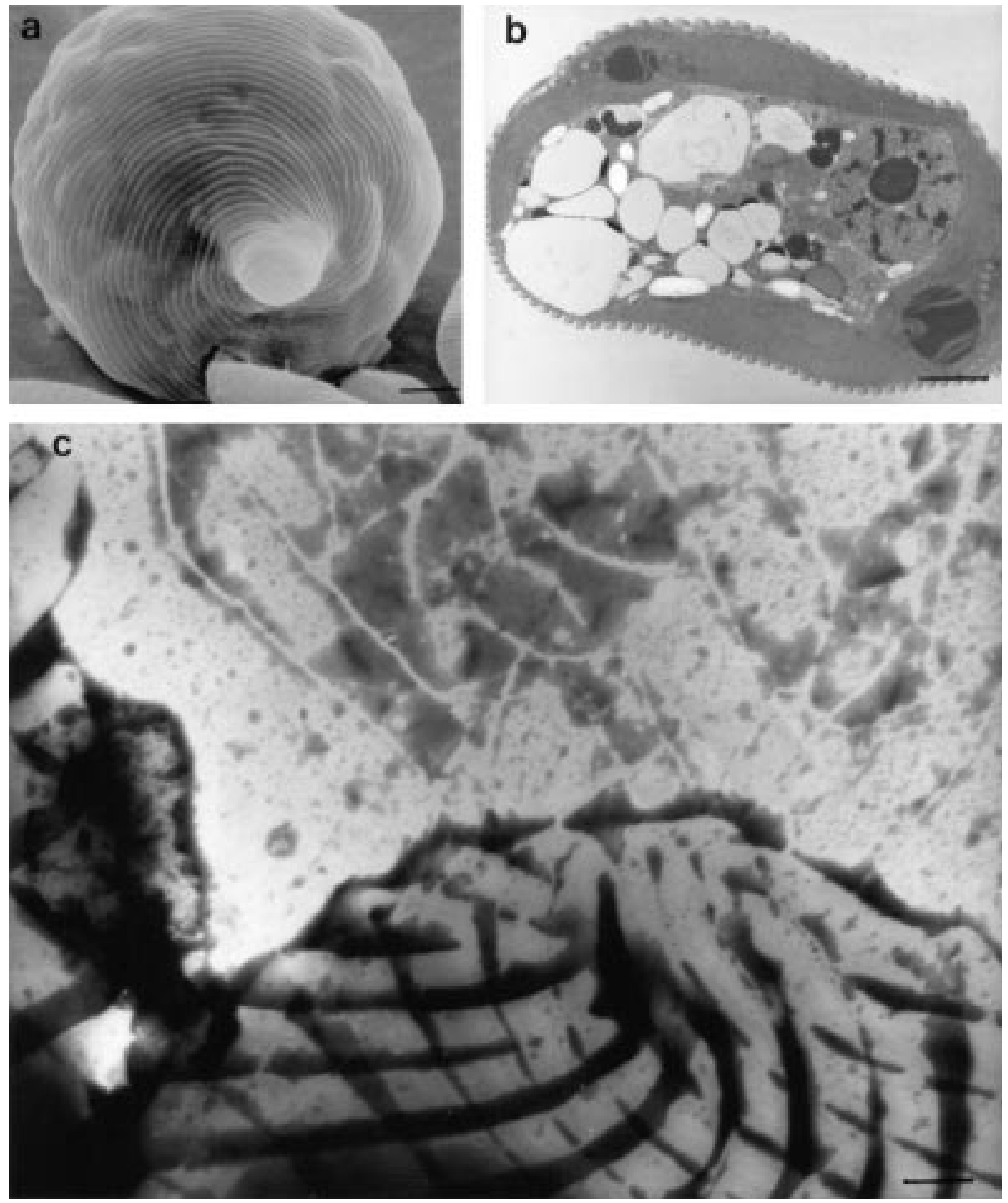

Fig. 1. E. pailasensis sp. nov. A. Micrografía Electrónica de Barrido (MEB). Se observan los mionemas bordeando todo el organismo (barra $=1.4 \mu \mathrm{m}$ ), B. MET. Corte longitudinal. Se observa el núcleo, los cloroplastos con pirenoides, paramilón y otras organelas (barra $=3 \mu \mathrm{m}$ ). C. Micrografía Electrónica de Transmisión (MET). Tinción negativa mostrando la distribución de los mionemas y la presencia de filamentos saliendo de los mismos (barra= $0.4 \mu \mathrm{m}$ ).

Fig. 1. E. pailasensis sp. nov. A. SEM. The mionemas skirting the whole organism (scale $=1.4 \mu \mathrm{m}$ ), B. TEM. Longitudinal section. The nucleus, cloroplasts with pirenoid, paramylon and other organels. (scale $=3 \mu \mathrm{m}$ ). C. TEM. Negative staining showing the distribution of the mionemas and the presence of filaments leaving of the mionemas (scale $=0.4 \mu \mathrm{m})$. 
deposited in EMBL/GenBank with the accession number AJ312953, demonstrated a 70\% of the identity with that of E. gracilis (Sittenfeld et al. 2002).

\section{AGRADECIMIENTOS}

Los autores agradecen al Area de Conservación Guanacaste, la Agencia Española de Cooperación Internacional (AECI), la Vicerrectoría de Investigación de la Universidad de Costa Rica y a Francisco Hernández-Chavarría por sus comentarios y sugerencias.

\section{RESUMEN}

Se describe una nueva especie de euglena aislada de las Pailas de Barro Caliente del Volcán Rincón de la Vieja, Costa Rica. Esta especie se caracteriza por habitar sitios ácidos y calientes. Euglena pailasensis sp. nov. tiene como características principales: la ausencia de flagelos, presencia de filamentos similares a "pilis", presencia de cloroplastos con pirenoides atravesados por varios tilacoides, además, es termotolerante y acido-tolerante. Los análisis filogenéticos para el gen ADNr $18 \mathrm{~S}$ y la secuencia del gen para la enzima Gap C indican que la nueva especie está relacionada con E. mutabilis. Las características taxonómicas basadas en la morfología, biología y secuencia del ADNr 18S y los genes GAP C, son discutidas y comparadas con otras especies relativamente cercanas al género.

\section{REFERENCIAS}

Sittenfeld A., M. Mora, J.M. Ortega, F. Albertazzi, A. Cordero, M. Roncel, E. Sánchez, M.Vargas, M. Fernández, J. Weckesser \& A. Serrano. 2002. Characterization of a photosynthetic Euglena strain isolated from acidic hot mud pool of a volcanic area of Costa Rica. FEMS Microbiology Ecology. 42: 151-161.

Sánchez E., M. Vargas, M. Mora, J.M. Ortega, A. Serrano, E. Freer \& A. Sittenfeld. 2004. Descripción ultraestructural de Euglena pailasensis (Euglenozoa) del Volcán Rincón de la Vieja, Guanacaste, Costa Rica. Rev. Biol. Trop. 52: 31-40.

Vismara R., L. Barsanti, P. Lupetti, V. Passarelli, D. Mercati, F. Dallai \& P. Gualtieri. 2000. Ultrastructure of the pellicle of Euglena gracilis. Tissue \& Cell 32(6): 451-456. 